\title{
White-light oscillations during a flare on II Peg
}

\author{
M. Mathioudakis ${ }^{1}$, J. H. Seiradakis ${ }^{2}$, D. R. Williams ${ }^{3}$, S. Avgoloupis ${ }^{2}$, D. S. Bloomfield ${ }^{1}$, and R. T. J. McAteer ${ }^{1}$ \\ 1 Department of Pure and Applied Physics, Queens University Belfast, Belfast BT7 1NN, Northern Ireland, UK \\ 2 University of Thessaloniki, Department of Physics, Section of Astrophysics Astronomy and Mechanics, \\ 54124 Thessaloniki, Greece \\ 3 Mullard Space Science Laboratory, University College London, Holmbury St Mary, Dorking, Surrey, RH5 6NT, UK
}

Received 14 January 2003 / Accepted 11 March 2003

\begin{abstract}
We analyse the intensity oscillations observed in the gradual phase of a white-light flare on the RS CVn binary II Peg. Fast Fourier Transform power spectra and Wavelet analysis reveal a period of $220 \mathrm{~s}$. The reliability of the oscillation is tested using several criteria. Oscillating coronal loop models are used to derive physical parameters such as temperature, electron density and magnetic field strength associated with the coronal loop. The derived parameters are consistent with the nearsimultaneous X-ray observations of the flare. There is no evidence for oscillations in the quiescent state of the binary.
\end{abstract}

Key words. waves - stars: flare - stars: binaries: spectroscopic - stars: oscillations - stars: magnetic fields

\section{Introduction}

Since the discovery of the solar five minute oscillation in the 1960 s, there is ample observational evidence for waves throughout the solar atmosphere (Stein \& Leibacher 1974). Wave dissipation is one of the mechanisms considered for energy input to the solar atmosphere with strong evidence for coupling between waves at the solar surface and magnetic structures in the transition region and corona. Their oscillatory signatures can be seen in imaging observations, Doppler shifts and intensity variations (Aschwanden et al. 2001). There is evidence that the excitation of waves in the upper atmosphere is often associated with reconnection events. For example, Kane et al. (1983) observed large amplitude intensity pulsations with a period of $8 \mathrm{~s}$ in hard X-rays and microwaves. The first evidence for spatial oscillations in coronal loops (Fe IX, $10^{6} \mathrm{~K}$ ) was presented by Aschwanden et al. (1999) where the $280 \mathrm{~s}$ period is suggestive of a resonant coupling with the photosphere. In both cases the oscillations were associated with solar flares.

In the stellar case, Rodonò (1974) reported white light intensity oscillations (13 s) during a flare on II Tau. Mullan (1976) attributed these to electron precipitation triggered by electron cyclotron waves propagating between starspots at the opposite poles of the star. Houdebine et al. (1993) observed variations (160 s) in the centroids of the Ca II H\&K lines, that were interpreted in terms of periodic motions in a prominence triggered by a flare disturbance.

Here we report intensity oscillations detected in the Johnson $U$-band during a flare on the RS CVn binary II Peg.

Send offprint requests to: M. Mathioudakis, e-mail: M.Mathioudakis@qub.ac.uk
The observations were carried out at Stephanion Observatory, Greece, in August 1989 using the 0.75 m Cassegrain reflector of the University of Thessaloniki, as part of a multiwavelength campaign on the binary. The energy of the flare event in optical wavelengths was in excess of $10^{36}$ ergs. The physical parameters of the flare have been described in detail by Doyle et al. (1991, 1992). The present paper will focus on the intensity variations observed in the gradual phase of the event. In Sect. 2 we present the methods used for the analysis of the observations and the search for reliable periodicities. In Sect. 3 we discuss our results in the context of oscillating coronal loop models and derive the physical parameters of the loop. Concluding remarks are given in Sect. 4.

\section{The search for intensity oscillations}

The impulsive phase of the flare is followed by a series of intensity variations (Fig. 1). These intensity variations were analysed by Fast Fourier Transform (FFT) and Wavelet analysis. While a FFT searches for periodic signatures with a basic exponential function and decomposes the signal into infinite length of sines and cosines, the analysing Wavelet is a time localised oscillatory function continuous in both frequency and time. It is therefore suited for the identification of transient oscillations. The Wavelet used in this study is the product of a Gaussian with a sinusoidal function and is known as the Morlet Wavelet (Torrence \& Compo 1998). In Fig. 2b we show the Wavelet power transform of the time series immediately after the impulsive phase of the event. The cone of influence (COI), crosshatched area in the plot, defines the area in the Wavelet diagram where edge effects become important. Any frequencies within the COI are disregarded. 


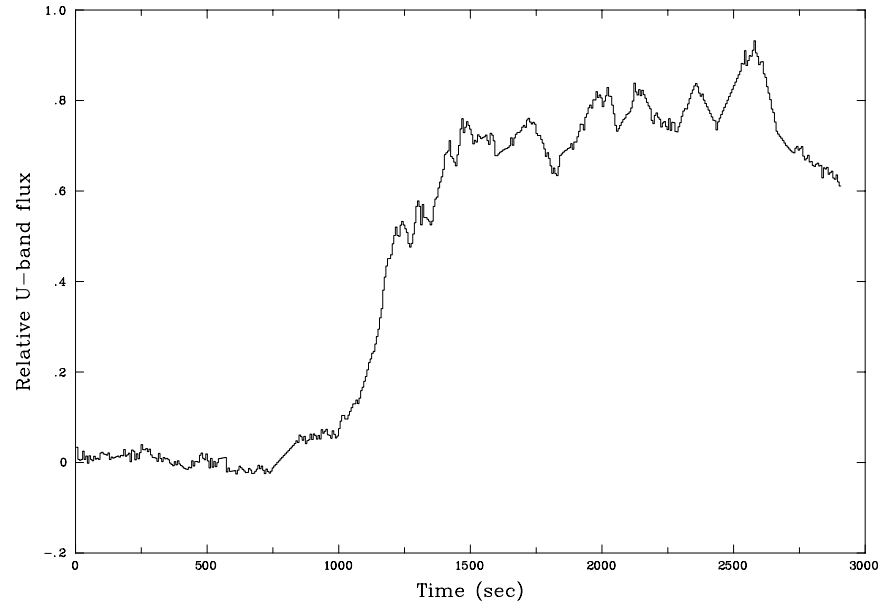

Fig. 1. $U$-band lightcurve of the II Peg flare. Time is given in seconds since 01:30 UT of 17 August 1989.

We use a number of criteria to examine the reliability of any oscillatory signature. The first is a test against spurious detections of power that may be due to Poisson noise. If a peak in the power spectrum is above the background, it can be a true feature at a given confidence level. The time series is assumed to be normally distributed, consistent with photon noise, and to follow a $\chi^{2}$ distribution with two degrees of freedom. The $99 \%$ confidence level is calculated by multiplying the power in the background spectrum by the values of $\chi^{2}$ corresponding to the 99th percentile of the distribution. The areas where power at the $99 \%$ confidence level (or higher) has been detected are indicated by the contours in Fig. 2b. The Wavelet power transform summed over time and the Fast Fourier power spectrum are shown in Fig. 2c.

In the second criterion the time-series is compared with a large number (1500) of randomised time-series with an identical distribution of counts (see Banerjee et al. 2001). By comparing the number of times that the power transform of the random series will produce a peak of equal or greater power than the peak power found in the input data, the confidence level of each peak at each timestep is calculated. A high value for the probability determined $(p)$, implies that there is no periodic signal in the data while a low value suggests that the detected periodicity is real. The probability levels $(1-p) \times 100$ for our time-series are shown in Fig. 2 d.

The third criterion guards against random, pulse-like variations in the data which might be treated as real. We only consider as reliable those detections that last for at least three periods. Our analysis reveals a reliable periodicity at approximately $220 \mathrm{~s}$. A sinusoidal signal with a period of $220 \mathrm{~s}$ is superimposed on the data (Fig. 2a). The above criteria were also applied to the pre-flare quiescent state of II Peg. We find no evidence for periodicities in the quiescent state of the binary.

\section{Discussion}

The white-light flux that we see in the $U$-band is due to continuum emission with some contribution from the higher Balmer lines and $\mathrm{Ca}$ II $\mathrm{H} \& \mathrm{~K}$. It is therefore, mainly of photospheric
Table 1. Physical parameters of the II Peg flare.

\begin{tabular}{lll}
\hline \hline & X-rays $^{a}$ & $U$-band $^{b}$ \\
\hline$T(\mathrm{~K})$ & $6.2 \times 10^{7}$ & $2 \times 10^{8}$ \\
$n_{e}\left(\mathrm{~cm}^{-3}\right)$ & $6.4 \times 10^{11}$ & $4 \times 10^{11}$ \\
$B$ (Gauss) & $525^{c}$ & 600 \\
\hline
\end{tabular}

${ }^{a}$ : Derived from the X-ray model of Doyle et al. (1992).

${ }^{b}$ : Derived from the observed period and amplitude of the oscillation and the model of Zaŭtsev \& Stepanov (1989).

${ }^{c}$ : Minimum value for the coronal field strength.

origin with a minor chromospheric contribution. The $220 \mathrm{~s}$ oscillation that followed the impulsive phase could either be triggered by the flare or be a property of the flaring region itself. The extensive analysis of the X-ray properties of the II Peg flare, carried out by Doyle et al. (1992), revealed physical parameters similar to those found in solar two-ribbon flares. These parameters, listed in Table 1, were derived primarily from the start of the decay phase. The first X-ray point in the lightcurve was obtained approximately five minutes after the last $U$-band observation. Assuming an aspect ratio of 0.25 (often seen in solar two ribbon flares), a loop length of $L \approx$ $5 \times 10^{10} \mathrm{~cm}$ can be derived. This value will be used in the following analysis.

The periodicities observed in some solar X-ray flares have often been interpreted in terms of Alfvèn oscillations of a coronal loop (Zaĭtsev \& Stepanov 1989). It is suggested that these oscillations arise from the stream of high energy particles that appear during the impulsive phase of the event. Zaĭtsev \& Stepanov (1989) derive the following relations between flare loop parameters such as temperature, density and magnetic field strength as a function of loop length, period and amplitude of the oscillation.

$T \sim 1.2 \times 10^{-8} \frac{L^{2}}{\tau^{2}} \frac{\Delta I}{I} \mathrm{~K}$

$n_{\mathrm{e}} \sim 2.2 \times 10^{-10} \frac{L^{3}}{Q \tau^{4}}\left(\frac{\Delta I}{I}\right)^{1.5} \mathrm{~cm}^{-3}$

$B \sim 6.7 \times 10^{-17} \frac{L^{2.5}}{Q^{0.5} \tau^{3}}\left(\frac{\Delta I}{I}\right)^{0.75}$ Gauss

where $T, n_{\mathrm{e}}, L, B$ are the temperature, density, length and magnetic field strength of the loop. Our oscillation has a period $\tau=220 \mathrm{~s}$, amplitude $\frac{\Delta I}{I} \approx 0.3$ and a quality factor $Q=6$. The quality factor is determined from the number of individual flare bursts. Substituting these values into Eqs. (1-3) we derive the parameters of the flare loop listed in the $U$-band column of Table 1. The Zaitsev \& Stepanov (1989) model has also been used for the analysis of the transient periodicities of active red dwarf stars (Mullan et al. 1992).

Our temperature estimate is somewhat higher than the estimate of Doyle et al. (1992). However, their analysis is based on the decay phase of the lightcurve while our estimates refer to the gradual phase. Temperatures of the order of $10^{8} \mathrm{~K}$ are quite common in the flaring coronae of active cool stars. For example, Stern et al. (1992) derived a temperature of $7 \times 10^{7} \mathrm{~K}$ 


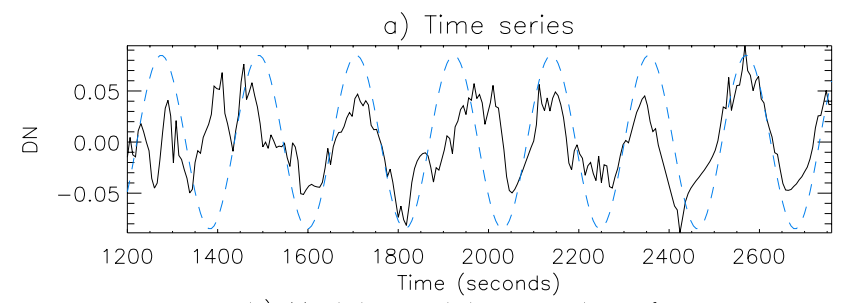

c) Fourier and

b) Morlet wavelet power transform Global Spectra
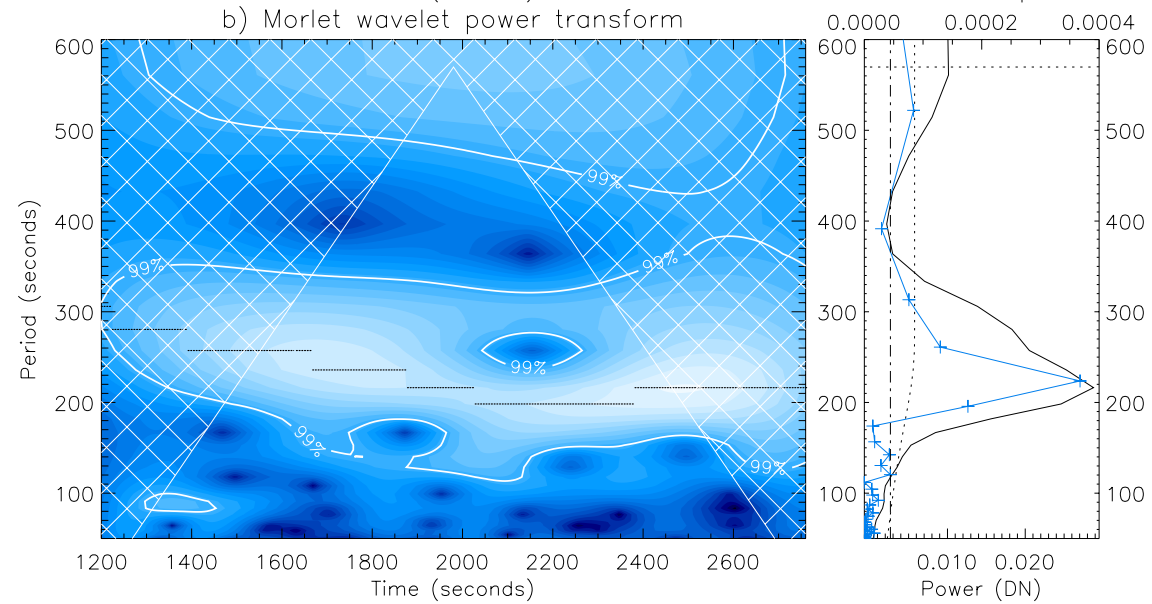

d) Confidence level for peak power

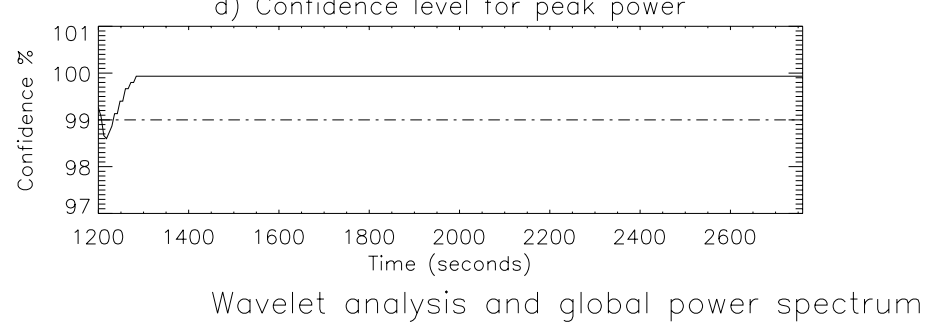

Fig. 2. a) The filtered timeseries used for our analysis b) The Morlet wavelet power transform of the post-impulsive phase part of the August 1989 II Peg flare. Period is plotted as a function of time from the begining of the entire time series. Contours are computed by defining the power, at each period, at which detections are $99 \%$ confident using a $\chi^{2}$ distribution with two degrees of freedom. c) Summation of the wavelet power transform over time (full line) and the Fast Fourier power spectrum (crosses) over time, plotted as a function of period. Both methods have detected a well pronounced 220 s oscillation. The global Wavelet (dotted line) and Fourier (dashed dotted line) $99 \%$ significance levels are also plotted. Periods above the horizontal line (dotted) fall within the COI. d) The confidence levels for the frequency that contains the greatest power, dark lines in $\mathbf{b}$ ), are plotted as a function of time (full line).

during a flare on Algol, while Pan et al. (1997) find $10^{8} \mathrm{~K}$ during a flare on EQ1839.6+8002. There is ample evidence for temperatures in excess of $10^{7} \mathrm{~K}$ in the quiescent state of II Peg (Griffiths \& Jordan 1998). As the II Peg flare is one of the most energetic events observed to date, temperatures of $10^{8} \mathrm{~K}$ are not surprising.

Nakariakov \& Ofman (2002) have developed a method for determining the magnetic field strength $(B)$ of coronal loops that are oscillating as a result of standing kink mode waves. The magnetic field strength can be determined from the period of the oscillation, loop length and mass density inside $\left(\rho_{0}\right)$ and outside $\left(\rho_{\mathrm{e}}\right)$ the loop using the following relation:

$B=\frac{\sqrt{8 \pi} L}{\tau} \sqrt{\rho_{0}\left(1+\frac{\rho_{\mathrm{e}}}{\rho_{0}}\right)}$

For the density outside the loop we use a value of $n_{\mathrm{e}}=1 \times 10^{11} \mathrm{~cm}^{-3}$. This value was derived from EUVE observations of Fe XXI for the quiescent corona of II Peg (Griffiths $\&$ Jordan 1998). For the density inside the loop we use the value of $n_{0}=6.4 \times 10^{11} \mathrm{~cm}^{-3}$ of the flare (see Table 1). Substituting the values into the above relation we derive a magnetic field strength of $\approx 1200 \mathrm{G}$. Note that the equation presented in Nakariakov \& Ofman (2001) requires a correction factor of 0.64 .

\section{Conclusions}

We report a $220 \mathrm{~s}$ intensity oscillation observed in the gradual phase of a white-light flare on II Peg. The oscillation could be triggered by the flare or be a property of the flaring loop itself. The parameters of the oscillation are combined with coronal loop models, to determine the magnetic field strength and temperature of the loop. The derived values, although quite high, are expected given the energetics of the flare. They are also consistent (to within a factor of 2) with an independent modelling of the X-ray data.

We have applied the Nakariakov \& Ofman coronal seismology technique, developed for the solar corona, on this stellar 
oscillation. We find no evidence for periodicity in the pre-flare quiescent state of the binary.

Acknowledgements. This work was supported by the UK Particle Physics and Astronomy Research Council and the Northern Ireland Department of Employment and Learning. MM would like to thank Drs. D. Jevremovic and V. Nakariakov for useful discussions. Wavelet software was provided by C. Torrence and G. Compo and is available at URL: http://paos . colorado. edu/research/wavelets/

\section{References}

Aschwanden, M. J., Fletcher, L., Schrijver, C. J., \& Alexander, D. 1999, ApJ, 517, 977

Aschwanden, M. J., Poland, A. I., \& Rabin, D. M. 2001, ARA\&A, 39, 175

Banerjee, D., O’Shea, E., Doyle, J. G., \& Goossens, M. 2001, A\&A, 371,1137

Doyle, J. G., Kellett, B. J., Byrne, P. B., et al. 1991, MNRAS, 248, 503
Doyle, J. G., van den Oord, G. H. J., \& Kellett, B. J. 1992, A\&A, 262, 533

Griffiths, N. W., \& Jordan, C. 1998, ApJ, 497, 883

Houdebine, E. R., Foing, B. H., Doyle, J. G., \& Rodono, M. 1993, A\&A, 274, 245

Kane, S. R., Kai, K., Kosugi, T., et al. 1983, ApJ, 271, 376

Mullan, D. J. 1976, ApJ, 204, 530

Mullan, D. J., Herr, R. B., \& Bhattacharyya, S. 1992, ApJ, 391, 265

Nakariakov, V. M., \& Ofman, L. 2001, A\&A, 372, L53

Nakariakov, V. M., \& Ofman, L. 2002, in Solar variability: from core to outer frontiers, ed. A. Wilson, ESA SP-506, 461

Rodonò, M. 1974, A\&A, 32, 337

Pan, H. C., Jordan, C., Makishima, K., et al. 1997, MNRAS, 285, 735

Stein, R. F., \& Leibacher, J. 1974, ARA\&A, 12, 407

Stern, R. A., Uchida, Y., Tsuneta, S., \& Nagase, F. 1992, ApJ, 400, 321

Torrence, C., \& Compo, G. P. 1998, Bull. Amer. Meteor. Soc., 79, 61

Ză̌tsev, V. V., \& Stepanov, A. V. 1989, Sov. Astron. Lett., 15, 66 